\title{
DESAFIOS DE TRATAMENTOS PARA DEPENDENTES QUÍMICOS
}

Asllen Leonardo Delgado Longo, Regiane Oliveira da Silva, Danielle Yurie Moura da Silva.

Universidade do Oeste Paulista, UNOESTE, Curso de Direito, PRESIDENTE PRUDENTE, SP. E-mail:

leold193@hotmail.com; regiane-olis@hotmail.com

\section{RESUMO}

O estudo tem por objetivo explorar os desafios encontrados em tratamentos realizados por dependentes químicos e as consequências que este fenômeno causa no meio jurídico, regido pela Lei $\mathrm{N}^{\circ} 11.343 / 06$, que é o Sistema Nacional de Políticas Públicas sobre Drogas, como forma de prevenção do uso indevido e também de usuários e dependentes de drogas não autorizados, as dificuldades encontradas são as intrínsecas e extrínsecas ao longo dos tratamentos. A metodologia aplicada foi hipotético-dedutivo, legislação, doutrinas e livros pertinentes ao tema. Assim, a voluntariedade do dependente é um dos principais problemas encontrados, más a persistência da família em leva-lo a pessoas capacitadas e mostrar os resultados adquiridos de outros dependentes, para que possam se espelhar e adquirir força de vontade para mudar seus comportamentos, fazendo uma reversão na situação em que se encontrava. Já no meio jurídico e público é esperado novos projetos de acolhimentos e profissionais qualificados.

Palavras-chave: DESAFIOS. TRATAMENTOS. CONSEQUÊNCIAS. VOLUNTARIEDADE. DEPENDENTES.

\section{CHEMICAL DEPENDENT TREATMENT CHALLENGES}

\section{ABSTRACT}

The study aims to explore the challenges encountered in treatments performed by drug addicts and the consequences that this phenomenon causes in the legal environment, governed by Law No. 11,343 / 06, which is the National System of Public Policies on Drugs, as a form of prevention of undue use and also of users and dependents of unauthorized drugs, the difficulties encountered are intrinsic and extrinsic throughout the treatments. The applied methodology was hypotheticodeductive, legislation, doctrines and books pertinent to the theme. Thus, dependency voluntariness is one of the main problems encountered, as well as the persistence of the family in taking it to trained people and showing the acquired results of other dependents, so that they can mirror and acquire the willpower to change their behaviors. a reversal in the situation in which it was. In the juridical and public environment, new projects of qualified professionals and professionals are expected.

Keywords: CHALLENGES. TREATMENTS. CONSEQUENCES. VOLUNTARINESS. DEPENDENTS. 


\section{INTRODUÇÃO}

Em relação aos dependentes químicos, muito se discute questões relacionadas aos entorpecentes, mais conhecidos como drogas, onde grande parte delas é produzida a partir de plantas e em laboratórios. Essas substâncias causam dependência química ou psicológica, perante o ordenamento jurídico ou cultural, podendo levar a óbito o indivíduo que há consome, regido pela Lei de Dependentes Químicos ou Usuários, que confirmou a obrigação de uma ação penal para aqueles que praticarem o ato. Esses produtos acabam fazendo com que usuários tenham uma vida mais complicada, sem perspectiva de futuro e de evolução profissional. Um indivíduo que venha a desenvolver a dependência química necessita de tratamento e principalmente de apoio de seus familiares, para que o mesmo consiga se reestabelecer e se curar do vicio. John Elliot Burns é autor do livro "O Caminho dos Doze Passos", que retrata doze passos de um tratamento para a recuperação da dependência química. Além de escritor ele foi um dos pioneiros no estudo e no trabalho com dependentes químicos no Brasil.

A influência das drogas vinculadas à ação do aborto que é tratada pelos artigos 124 a 128 do Código Penal relata as formas e em quais situações ele é ou não permitido. Além disso, assuntos relacionados diretamente a ação do aborto, questão essa que é discutida frequentemente na mídia em geral e outros problemas decorrentes da dependência química e do inicio dos tratamentos como: violência, alucinações, transtornos mentais e outros problemas que podem estimular resultados não desejados.

Assim, a ocorrência de problemas paralelos à doença torna o objetivo do estudo muito mais sólido, pois esses "problemas" serão tratados como desafios de tratamentos para que os dependentes químicos possam ir melhorando gradativamente até chegar ao ponto de estarem curados, tudo pela total dedicação e persistência para ultrapassar esses desafios.

\section{METODOLOGIA}

O procedimento aplicado foi à consulta em livros e doutrinas pertinentes ao tema proposto, como forma de explorar conteúdos, aplicando-se assim o método hipotético-dedutivo para compor ideias que atinjam metas e propostas através dos conteúdos adquiridos.

\section{DEPENDENTES QUÍMICOS: INFORMAÇÕES GERAIS}

De acordo com a Organização Mundial da Saúde (OMS, 2017), "droga é qualquer substância que interfere no funcionamento de um ou mais de seus sistemas". Ela (2016), também define a dependência química como "uma doença crônica, progressiva, ou seja, que piora com o passar do tempo, primária, que gera outras doenças e fatal".

A dependência química é um transtorno mental caracterizado por um grupo de sinais e sintomas decorrentes do uso de drogas. Existem diversos relatos em relação à origem das drogas, um deles é de pesquisadores e colunistas do Portal Educação (2015) que tiveram suas primeiras aparições relatadas na época em que o Brasil ainda era uma Colônia, quando os índios utilizavam plantas e ervas tóxicas para seus rituais, convenções e manifestações religiosas. Com a utilização frequente dessas substâncias surgiu a titularização do dependente químico que é o individuo que utiliza frequentemente entorpecentes regulamentadas pela portaria 344/98 da ANVISA (Agência Nacional de Vigilância Sanitária, vinculada com o Ministério da Saúde). Como todos sabem, as drogas são muito prejudiciais à saúde, causando uma dependência no corpo humano de acordo com o período de utilização e quantidade, em regra no indivíduo que utiliza em longo prazo pode ocorrer: problemas na capacidade de pensar, rins, coração, fígado, pulmões e outros, levando por fim na maioria dos casos a morte.

\section{A INFLUÊNCIA DAS DROGAS NA SOCIEDADE}


Segundo Dias (2012), o grande aumento da utilização de drogas no Brasil ocorreu em 1960, principalmente da maconha, por isso o Brasil resolveu ingressar no cenário internacional de combate às drogas promulgando em 1964 uma Convenção Única sobre Entorpecentes, que no futuro veio a ser um Plano Estratégico de Combate as Drogas chamado de SISNAD (Sistema Nacional de Politicas Públicas sobre Drogas), por meio de um Decreto Legislativo. Como sabemos as drogas são encontradas nas sociedades, sejam elas licitas ou ilícitas, causando grandes transtornos tanto para o individuo que á usufrui quanto para a sociedade que recebe seus impactos.

No Brasil são diversos os problemas, sejam eles em relação à doença ou ao tratamento, pois para realizar os tratamentos visto que é um campo vasto de doenças que a dependência pode causar, faz com que envolvam muitos profissionais dificultando a acessibilidade do tratamento. Segundo o psiquiatra e consultor da Secretaria Nacional de Politicas sobre Drogas, José Manoel Bertolete (2011) diz que:

O sucesso de qualquer tratamento é a voluntariedade do dependente de se submeter às orientações feitas, sendo o primeiro passo, o afastamento em relação às drogas chamado período de desintoxicação, que tem o papel de reduzir os danos a que o usuário fica exposto, más isso é apenas um pequeno passo para o inicio do tratamento. Pois os responsáveis em garantir o tratamento por completo estão presentes em sistemas de apoio médico, psiquiátrico, social, familiar e psicológico.

A desintoxicação é um dos momentos mais difíceis que o dependente enfrentará durante o tratamento, pois ao se desvincular do entorpecente ao qual seu organismo estava ligado causará diversas outras doenças sendo elas psicológicas ou não, por isso a Clinica Vidas (2018) diz que, tratamentos podem variar de acordo com o tempo ao qual a pessoa leva para se afastar das drogas e para a recuperação de possíveis complicações ao longo do tratamento que é muito frequente, este tempo é relativo e vai de acordo com o quadro analisado pelos profissionais.

De acordo com o Dr. Arthur Frazão (2018) existem clínicas de internamento para dependentes químicos espalhadas por todo país e o tempo de internamento varia de 2 a 3 meses, podendo ser parcial (só durante o dia) ou integral, onde a pessoa apenas sai de lá quando estiver completamente recuperada.

A Organização Mundial da Saúde (2017) divulgou que cerca de (quinhentas mil pessoas) foram vítimas de morte por dependência química, deixando toda a população assustada pelo grande aumento deste número ao decorrer dos anos. E agora o que fazer para que esse número possa decair? Se continuar assim, quais serão as consequências? Será que existe algum culpado em relação a isso?... Eis aí que surgem diversos questionamentos perante a sociedade, e o governo juntamente com programas de saúde tentará conter esse número ao máximo! Já que esta causa é uma das que mais matam jovens nos dias de hoje.

\section{CONSUMO DE ENTORPECENTES: UMA QUESTÃO DE SAÚDE OU CRIMINAL?}

Hoje a questão do consumo de entorpecentes é encarada como uma questão criminal por esse motivo foi criado diversas leis como forma de regimento do consumo desses entorpecentes ilícitos.

Na sociedade em que vivemos muito se discute a questão do consumo das drogas, e como forma de saciar posições da sociedade elas foram divididas em duas espécies: as que podem ser comercializados legalmente (drogas lícitas) e outros em que seu consumo e aquisição seriam restritos (drogas ilícitas), as drogas ilícitas só podem ser consumidas, desde que não prejudiquem a sociedade, pois não há como privar uma pessoa de fazer mal a si mesma. Os familiares querem ver seus familiares (dependentes químicos) sendo tratados por profissionais adequados e por 
clinicas que o ajudem a se recuperar do vicio, pois a prisão não seria a forma adequada de melhorar esse problema, por isso seria a princípio é uma questão mais de saúde do que de justiça até o ponto em que esses indivíduos não violem direito de terceiros.

Esse é o certo a ser feito, porém não é a realidade do que acontece, visto que ao ser pego usando esse tipo de droga proibida as pessoas não tem a preocupação em ajudar e propor a ideia de tratamento, a primeira ação delas é aplicar a lei 11.343/06 para que o dependente seja punido e submetido ao castigo em vez de incentivá-lo ao tratamento que é o meio mais eficaz e humanístico, pois além de resolver o problema da dependência ele também ajuda a diminuir a frequência de outros crimes de serem realizados como: tráfico, aborto, depressão e outras doenças ou crimes que decorrem do uso desses entorpecentes ilícitos. Em âmbito social, Gilberto Velho (2008, p. 132), afirma:

A droga associou-se à arma, essa que é a grande questão. O tráfico de armas está associado ao tráfico de drogas. $O$ uso quase que pastoril da maconha, esse sentido que, embora não fosse ingênuo, porque na época isso era objeto de repressão, era proibido, era ilegal, mas era um tipo de protesto que se fazia num plano muito pacífico. Entretanto, de uns vinte e cinco, trinta anos para cá, com a proibição das drogas, e com as drogas se tornando mais conhecidas, se disseminando com a globalização, nós assistimos a montagem de uma gigantesca máquina criminosa que não é só de traficantes de drogas, é uma máquina criminosa que se não for lidar com droga vai lidar com outra coisa, se não é droga é arma, é arma junto com droga.

Muitos crimes são realizados em decorrência da dependência química e isso acaba causando um vicio, fazendo com que o ser humano realize cada vez mais crimes. Como forma de interceptar essas condutas lesivas para a sociedade esse dependentes devem ser submetidos a tratamentos de dependência química, excluindo também qualquer possibilidade de realização de crimes por influência da dependência. 0 Art. $28^{\circ}$, da Lei $11.343 / 06$, cuida da figura do usuário ou dependente de drogas, sendo instituída em sua regulamentação a medida educativa de comparecimento ao programa ou curso educativo, que assegura ao individuo exposto à dependência química seu devido tratamento.

\section{CONCLUSÃO}

$\mathrm{Na}$ atualidade, a dependência química é vista por leigos como uma doença ao qual sua punição seria resolvida com a prisão sendo ela comparada a um crime. Em todos os casos é de suma importância sabermos que isso está errado, pois o recurso adequado e indicado nos dias de hoje para esse tipo de doença é o tratamento.

Os problemas enfrentados pelos dependentes são tantos que acabam sendo retratados em livros, revistas, novelas, debates e outros meios como forma de expor suas dificuldades. Este problema não se passa somente no Brasil e sim no mundo todo, mas foram criadas diversas leis que proporcionam segurança e tratamentos. A Lei 10.216/2001 que abrange além de doentes mentais, usuários de drogas e álcool, servindo como um regimento de âmbito nacional junto com o Ministério da Saúde regido pela lei federal conhecida como Lei Manicomial, que proporciona a internação apenas em casos aos quais os recursos extra-hospitalares não forem suficientes.

Por outro lado, é dever do Estado zelar pela saúde de todos os cidadãos, visto que é garantido pela Constituição Brasileira esse princípio fundamental. Por esse motivo foram criados os Centros de Atenção Psicossocial (CAPS) como forma de atender os dependentes ou pacientes que apresentam vínculos com alguma substância prejudicial á saúde. 
Assim, as principais dificuldades de tratamento dos dependentes químicos não são as do mundo exterior e sim de seu próprio interior, pois antes de enfrentar o tratamento o paciente tem que se submeter a ele com sua própria força de vontade para que o primeiro passo do tratamento seja dado, fazendo com que o paciente saia da situação ao qual se encontrava e retorne a sua vida cotidiana.

\section{REFERÊNCIAS}

ABORDAGEM multidisciplinar para reabilitação de dependentes químicos. Jornal do Senado - Em Discussão. Brasília. 08 . Ago. 2011. / Disponível em: < https://www.senado.gov.br/noticias/Jornal/emdiscussao/dependencia-quimica/tratamento-paradependentes-quimicos/abordagem-multidisciplinar-reabilitacao-dependentes-quimicos.aspx >./ Acesso em: 26 jun. 2018.

ATAÍDE L. O Desafio de Tratamento de um Dependente Químico. Jornal da Cidade Online, São Paulo, 20 out. 2015./ Disponível em:< https://www.jornaldacidadeonline.com.br/noticias/1357/odesafio-do-tratamento-de-um-dependente-quimico >. Acesso em: 22 jun. 2018.

Beatriz, C. L. et. al.; Almeida, J. (Editor). Drogas e Cultura: Novas Perspectivas. $1^{\circ}$ ed. Salvador: Bahia, 2008. / Disponível em: file://C:/Users/USUARIO1/Downloads/Drogas\%20e\%20Cultura\%20(1).pdf >./Acesso em: 24 jul. 2018.

CLÍNICA VIDA: REABILITAÇÃO EM DEPENDÊNCIA QUÍMICA. Tratamento Contra Drogas: O Desafio para a Recuperação do Dependente Químico. Agencia MK10, 2018./ Disponível em: < https://www.institutovidas.org/single-post/2018/03/20/TRATAMENTO-CONTRA-DROGAS-

\%E2\%80\%93-O-DESAFIO-PARA-A-RECUPERA\%C3\%87\%C3\%830-DO-DEPENDENTE-

QU\%C3\%8DMICO >./ Acesso em: 25 jun. 2018.

Colunista Portal Educação. A Origem das Drogas na História e seu Surgimento no Brasil. Portal da Educação Tecnologia Educacional LTDA. / Disponível em:< https://www.portaleducacao.com.br/conteudo/artigos/medicina/a-origem-das-drogas-nahistoria-e-seu-surgimento-no-brasil/60298 >./ Acesso em: 30 jun. 2018.

Cynara, T. R.; Andréa, H. F. Tratamentos para Usuários de Drogas: Possibilidades, Desafios e Limites da Articulação entre as Propostas da Redução de Danos e da Psicanálise. Analytica: Revista de Psicanálise. São João Del Rei, v.2, n.2. Jun. 2013. / Disponível em: < http://pepsic.bvsalud.org/scielo.php?script=sci_arttext\&pid=S2316-51972013000100003>./ Acesso em: 22 jun. 2018.

Dr. Frazão A. Tipos, Efeitos e Consequências das Drogas para Saúde. Tua Saúde (Coor.). / Disponível em: < https://www.tuasaude.com/efeitos-das-drogas/ >./ Acesso em: 30 jun. 2018.

Dr. Frazão A. Tratamento para parar de usar Drogas. Tua Saúde (Coor.). / Disponível em: < https://www.tuasaude.com/tratamento-para-drogados/ >./ Acesso em: 30 jun. 2018.

Fuentes, L. Guerra às Drogas: Um Problema de Saúde Pública. Jornal da USP. São Paulo. 04. Jul. 2016. / Disponível em:< https://jornal.usp.br/atualidades/guerra-as-drogas-um-problema-desaude-publica/>./ Acesso em: 07 jul 2018. 
GUILHERME DA LUZ. Clínica Amigos em Mútua Ajuda. O Desafio para a Recuperação do Dependente Químico. Copyright, 2016/ Imultimídia. / Disponível em: < http://www.clinicaama.com.br/blog_ver.php?id=4>./ Acesso em: 22 jun. 2018.

Gouveia M. Tratamento de Dependentes Químicos é uma Discussão que precisa ir além das Páginas Policiais. Jornal Opção. Goiânia. 29. Nov. 2014. / Disponível em: < https://www.jornalopcao.com.br/reportagens/tratamento-de-dependentes-quimicos-e-umadiscussao-que-precisa-ir-alem-das-paginas-policiais-21951/ >./ Acesso em: 26 jun. 2018.

INSTITUTO BRASILEIRO DE CIÊNCIAS CRIMINAIS (Brasil). Presos Dependentes Químicos. Soares G. J. (aux. da seção de Pub. e Juris.). / Disponível em: < https://ibccrim.jusbrasil.com.br/noticias/2901389/presos-dependentes-quimicos >./ Acesso em: 26 jun. 2018.

Íntegra Comunicação Estratégica. Dependência Química é Considerada Transtorno Mental, Alerta Psiquiatra. 2016. Unimed Cuiabá. / Disponível em:< https://www.unimed.coop.br/web/cuiaba/noticias/dependencia-quimica-e-consideradatranstorno-mental-alerta-psiquiatra >./ Acesso em: 21 jul. 2018.

Ivo P. T. Droga é Questão de Saúde, não de Polícia, diz Professor de Direito da USP. Folha de São Paulo. / Disponível em: < https://www1.folha.uol.com.br/cotidiano/2015/06/1645395-droga-equestao-de-saude-nao-de-policia-diz-professor-de-direito-da-usp.shtml >./ Acesso em: 07 jul 2018.

Leal, J. J. Política criminal e a lei № 11.343/2006: Nova lei de Drogas, novo Conceito de Substância causadora de Dependência. Jus Navegandi. Teresina, v.1, n.1177, set. 2006. / Disponível em: < https://jus.com.br/artigos/8957/politica-criminal-e-a-lei-n-11-343-2006-nova-lei-de-drogas-novoconceito-de-substancia-causadora-de-dependencia >./ Acesso em: 26 jun. 2018.

MOVIMENTO SAÚDE UNIMED (CUIABÁ). Dependência Química é Considerada Transtorno Mental, Alerta Psiquiatra. Olhar Conceito. Cuiabá. 11. Fev. 2016. / Disponível em:< http://www.olhardireto.com.br/conceito/colunas/exibir.asp?id=635\&artigo=dependenciaquimica-e-considerada-transtorno-mental-alerta-psiquiatra >./ Acesso em: 08 set. 2018.

Organização Mundial de Saúde (Brasil). OMS afirma que Consumo de Drogas causa 500 mil Mortes Anuais. Agencia EFE. Globo Comunicação e Participações S.A. / Disponível em:< https://g1.globo.com/bemestar/noticia/oms-afirma-que-consumo-de-drogas-causa-500-milmortes-anuais.ghtml >./ Acesso em: 04 jul. 2018.

Queiroz G. USUÁRIO DE DROGAS: Mudança na lei de drogas no 11.343/2006. Portal Da Educação Tecnologia Educacional LTDA. / Disponível em:< https://www.portaleducacao.com.br/conteudo/artigos/idiomas/usuario-de-drogas-mudanca-nalei-de-drogas-n-11343-2006/55651 >./ Acesso em: 30 jun. 2018. 